\section{RMD Open}

Rheumatic \&

Musculoskeletal Diseases

\title{
Trajectories of fatigue in actively treated patients with established rheumatoid arthritis starting biologic DMARD therapy
}

\author{
Sella Aarrestad Provan (D), ${ }^{1}$ Brigitte Michelsen (D), ${ }^{1,2}$ Joseph Sexton, ${ }^{1}$ \\ Tillmann Uhlig, ${ }^{1}$ Hilde Berner Hammer ${ }^{1}$
}

To cite: Provan SA, Michelsen B, Sexton J, et al. Trajectories of fatigue in actively treated patients with established rheumatoid arthritis starting biologic DMARD therapy. RMD Open 2020;6:e001372. doi:10.1136/rmdopen-2020001372

- Supplemental material is published online only. To view please visit the journal online (http://dx.doi.org/10.1136/rmdo pen-2020-001372)

Received 26 June 2020 Revised 4 September 2020 Accepted 1 November 2020

Check for updates

(C) Author(s) (or their employer(s)) 2020. Re-use permitted under CC BY-NC. No commercial re-use. See rights and permissions. Published by BMJ.

${ }^{1}$ Department of Rheumatology, Diakonhjemmet Hospital, Oslo, Norway

${ }^{2}$ Department of Rheumatology, Hospital of Southern Norway Trust Kristiansand, Kristiansand, Norway

Correspondence to Sella Aarrestad Provan; sellaprovan@gmail.com

\section{ABSTRACT}

Objectives To define fatigue trajectories in patients with rheumatoid arthritis (RA) who initiate biological DMARD (bDMARD) treatment, and explore baseline predictors for a trajectory of continued fatigue.

Methods One-hundred and eighty-four patients with RA initiating bDMARDs were assessed at 0, 1, 2, 3, 6 and 12 months. Swollen and tender joint counts, patient reported outcomes (PROMs), blood samples and ultrasound examinations were collected at each time point. Fatigue was assessed by the fatigue Numeric Rating Scale $(0-10)$ from the Rheumatoid Arthritis Impact of Disease (RAID) questionnaire. Clinically significant fatigue was predefined as fatigue $\geq 4$. Three trajectories of interest were defined according to level of RAID fatigue: no fatigue ( $\leq 3$ at $5 / 6$ visits), improved fatigue ( $\geq 4$ at start, but $\leq 3$ at follow-up) and continued fatigue ( $\geq 4$ at $5 / 6$ visits). Baseline variables were compared between groups by bivariate analyses, and logistic regression models were used to explore baseline predictors of continued vs improved fatigue.

Results The majority of patients starting bDMARD therapy followed one of three fatigue trajectories, (no fatigue; $n=61$, improved; $n=33$ and continued fatigue; $n=53$ ). Patients with continued fatigue were more likely to be anti-citrullinated protein antibody and/or rheumatoid factor positive and had higher baseline PROMs compared to the other groups, while there were no differences between the groups for variables of inflammation including. Patient global, tender joint count and anxiety were predictors for the continued fatigue trajectory.

Discussion A trajectory of continued fatigue was determined by PROMs and not by inflammatory RA disease activity.

\section{INTRODUCTION}

Rheumatoid arthritis (RA) is an inflammatory joint disease that can potentially affect all synovial joints, causing joint destruction and disability. ${ }^{1}$ Fatigue is a common complaint among patients with $\mathrm{RA}$ and has a major impact on the burden of disease. In a recent Danish study, $61 \%$ of the
Key messages

What is known?

- Fatigue in RA is known to be multifactorial.

What this study adds?

- The longitudinal course of fatigue of the majority of patients with longstanding RA who start bDMARD therapy could be classified into three trajectories.

- Only $18 \%$ of patients starting a bDMARD were categorised into the improved fatigue trajectory, in this heterogeneous cohort.

- The trajectory of continued fatigue was predicted by PROMs and not by inflammatory RA disease activity or ultrasonographic joint evaluation.

How this impact on clinical practice?

- Patients who experience improved fatigue most frequently did so within the first months after the start of bDMARD therapy.

- PROMS are important determinants of continued fatigue and should be the focus of future interventions targeting this symptom.

patients with RA reported having moderate to severe fatigue. ${ }^{2}$ Fatigue was identified by patients with RA as an important patientreported outcome measure (PROM) during the development of the RA Impact of Disease (RAID) ${ }^{3}$ questionnaire. The aetiology of fatigue is multifactorial and is likely to be influenced both by disease-related inflammation, by pain, cognitive factors, depressive symptoms and personal issues in the individual patient's life. ${ }^{4}$

Disease activity in RA is assessed by composite measures of disease activity that usually include measures of swollen and tender joints, inflammation and patient-reported global assessment. Disease remission, at which disease activity falls to a pre-specified level, is the treatment target. RA is usually treated 
with conventional synthetic disease-modifying antirheumatic drugs (csDMARDs) from the time of diagnosis, and if remission is not reached within the desired time frame, dose escalation or change to a biologic DMARD (bDMARD) such as a TNFalpha-inhibitor (TNFi) is recommended. ${ }^{1}$

Relief of fatigue may improve quality of life, and treatment with TNFi has been shown to affect fatigue levels in a substantial proportion of patients. However, fatigue has been shown to persist at a clinically significant level in the majority of patients who are in remission. ${ }^{5}$ There is little knowledge regarding the factors predicting continued fatigue in patients treated with a bDMARD.

The objectives of this study were to define trajectories of fatigue in patients with RA who initiate bDMARD treatment and to define baseline predictors for continued fatigue.

\section{PATIENTS AND METHODS}

\section{Patients}

This study uses data from a previously described cohort of patients with RA fulfiling the American College of Rheumatology 1987 criteria $^{6}$ who were consecutively enrolled when they initiated or changed biologic DMARD treatment in the period from January 2010 to June $2013 .^{7}$ The patients were comprehensively assessed at baseline and after 1, 2, 3, 6, and 12 months. Patients who had answered the RAID questionnaire, including the question regarding fatigue, were selected for the current analyses. ${ }^{7}$ The study (Anzctr.org.au identifier ACTRN12610000284066) was approved by the Norwegian Regional Committee for Medical and Health Research Ethics South East (reference number 2009/1254) and the patients gave their written informed consent according to the Declaration of Helsinki.

\section{Clinical, laboratory and ultrasound assessments}

At each visit clinical examinations including examiner's global disease activity (0-100 VAS), 28 tender and swollen joint counts were performed by study nurses and blood samples were taken for laboratory assessments of erythrocyte sedimentation rate (ESR), (CRP) mg/L, Rheumatoid factor (RF) and anti-cyclic-citrullinated protein (CCP) antibodies. In addition, calprotectin $(\mu \mathrm{g} / \mathrm{L})$, a major leucocyte protein shown to be sensitive for rheumatic inflammation, was measured in EDTA plasma with an ELISA from CALPRO AS (Lysaker, Norway) according to the instructions of the manufacturer.

An experienced sonographer $(\mathrm{HBH})$ performed all the ultrasonography (US) examinations (using a dedicated Siemens Antares Excellence version machine, 5-13 MHz probe with PD frequency 7.3 MHz and PRF $391 \mathrm{~Hz}$, with no updates of the software during the study), blinded from the clinical assessments and laboratory markers from the same time points, as well as from previous US results. Grey scale (GS) ultrasound reflecting synovial hypertrophy and power Doppler (PD) ultrasound reflecting vascularity in the synovium, were scored semiquantitatively on a 4 -point scale $(0=$ no, $1=$ minor, $2=\bmod -$ erate, $3=$ major presence) of 36 joints (bilateral wrist (radiocarpal, midcarpal, radioulnar joints scored separately), metacarpophalangeal $1-5$, proximal interphalangeal 2-3, elbow, knee, ankle (tibiotalar), metatarsophalangeal 1-5) with the US atlas as reference, ${ }^{8}$ as well as four tendon sheaths (bilateral extensor carpi ulnaris and tibialis posterior). The sonographer has previously shown high reliability for US assessments of these joints and tendons. ${ }^{8}$

\section{Patient-reported outcome measures (PROMs)}

The patients were examined when initiating bDMARD therapy and assessed at baseline and after 1, 2, 3, 6 and 12 months. The PROMs included the RAID score, which has been developed in liaison with patients and includes fatigue, sleep, pain, physical function, physical and psychological well-being as well as coping, all scored on a 10 points numeric rating scale (NRS). ${ }^{3}$ The fatigue question is anchored at no fatigue and total exhaustion. In addition, patients scored their joint pain (0-100 visual analogue scale (VAS)), patient's global disease activity VAS (0-100), modified health assessment questionnaire (MHAQ 0-3, two main questions from the paincatastrophizing assessment ${ }^{7}$ and short form-36 mental health scale score (SF36MH). Anxiety and depression were assessed by the use of the Hospital Anxiety and Depression scale (HADS). The score of widespread pain was self-reported by indicating presence of pain at 19 prespecified sites during the past 7 days.

\section{Identification of fatigue trajectories}

Prior to data analyses, we had defined three trajectories to be of interest: no clinically significant fatigue, improved fatigue and continued clinically significant fatigue. Clinically significant fatigue was defined as RAID fatigue after discussions and a review of the literature $(\geq 4) .{ }^{9}$ The authors SAPR, TU and HBH examined the fatigue trajectory of each individual patient in line-plots. Using a visual data-driven approach we sought to categorise the maximum number of participants by examining changes in fatigue in relation to the cut-off over time while also constructing mutually exclusive categories (online supplemental table 1). We decided to place the cut-off between improved and permanent fatigue as change in RAID fatigue from $\geq 4$ to $\leq 3$ before the 5 th visit at 6 months. Patients who did not fit into the aforementioned trajectories were categorised as increasing fatigue' if there was no clinically significant fatigue at baseline but then a progression to clinically significant fatigue that persisted over several visits. Patients were categorised as 'no discernible pattern' if the fatigue level fluctuated over and below the cut-off for clinically significant fatigue, and as 'missing' if data was missing after the 2nd or 3rd visit. 


\section{Statistics}

Baseline variables were compared across groups using independent samples T-test, Wilcoxon rank-sum or $\mathrm{Chi}^{2}$ for normally distributed, non-parametric distribution or dichotomised data, respectively.

Logistic regression models were used to explore baseline predictors of continued vs improved fatigue. Each variable was entered into univariate models, adjusted for age and gender. Variables that predicted the outcome with a $\mathrm{p} \leq 0.1$ were entered into the multivariable model. Variables that were highly correlated (Pearsons correlation co-efficient $>0.7$ ) were examined in separate the multivariable models. Variables were removed according to level of significance (backward stepwise), until the final model only included significant predictors at $p<0.05$. Age and gender were forced into the model. Possible confounding was explored in the final model by re-entering the discarded variables to look for significance in the final model and examining the main effects for change in significance or a $25 \%$ or more change in the co-efficient.

Disease activity indicators and PROMs were plotted as estimated marginal means in figures across the trajectories, and differences between groups assessed by mixed models with maximum likelihood, adjusted for age, gender and time.

Last observation carried forward replaced missing data if data from one visit, but not the first visit, was missing ( $<5 \%$ of the different variables). Patients with $>1$ missing visit were not included in the analyses. All calculations were performed by the use of STATA version 14, and $\mathrm{p}<0.05$ was considered statistically significant.

\section{RESULTS}

Two hundred and eight patients had completed the RAID questionnaire for fatigue, 24 of these were missing from $>1$ visit and were excluded from the study.

Longitudinal trajectories of fatigue were examined in 184 patients (online supplemental table 1). The definition of three fatigue trajectories that allowed for the categorisation of the maximum number of patients into the three trajectories were: No fatigue (61 patients): fatigue score $\leq 3$ at baseline and at 4 out of 5 follow-up visits. Improved fatigue (33 patients): fatigue score $\geq 4$ at baseline but $\leq 3$ at the 6 - and 12-month visits. Continued fatigue (53 patients): fatigue level $\geq 4$ at 5 out of 6 visits. In addition, no discernible pattern was found in 34 patients and 3 patients had an increasing level of fatigue. The fatigue levels of four trajectories, adjusted for age and gender are presented in figure 1. 24 patients left the study after the second (4 patients) third (7 patients) or fourth visit (13 patients), these had comparable age, gender distribution and disease activity, to the participants remaining in the study. More details regarding the categorisation into trajectories are presented in the online supplemental table 1.

Baseline fatigue was significantly higher in the continued fatigue group compared to the no fatigue and

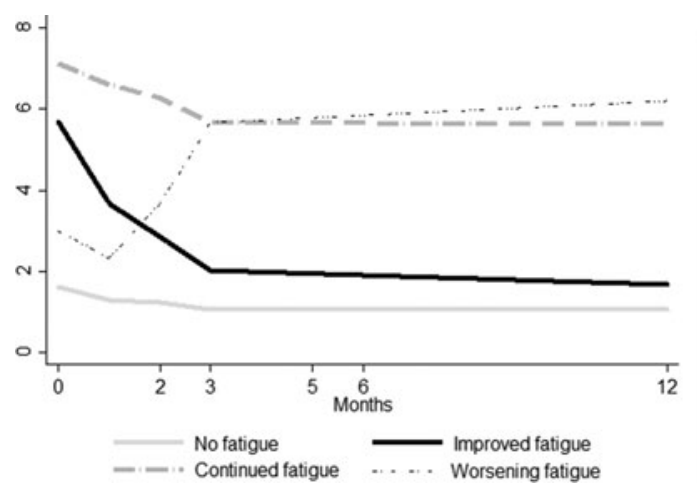

Figure 1 Longitudinal mean fatigue levels across trajectories of fatigue.

improved fatigue groups, and was also higher in the group with improved fatigue compared to no fatigue.

Table 1 describes demographics and clinical factors of the three groups. Patients with continued fatigue were more often female, were more likely to be anti-CCP and RF-positive, but had lower levels of education compared to patients with no clinically significant fatigue, and were more likely to be anti-CCP positive and have lower education than patients who had improved fatigue. Patients with continued fatigue had higher levels of all PROMs compared to patients with no fatigue, and also reported higher levels of most PROMs compared to patients who improved from their fatigue. Patients who had improved fatigue had higher levels of patient global, sleep disturbance, widespread pain and pain catastrophizing compared to patients with no fatigue. The average change (SD) in fatigue from baseline to 12-months was 0.49 (1.24) for the no-fatigue, 4.07 (0.28) for the improved fatigue and 1.49 (2.16) for the continued fatigue trajectory.

In logistic multivariable regression the trajectory of continued fatigue vs improved fatigue was predicted by baseline calprotectin, patient's global, tender joints and anxiety (table 2). The HADs anxiety and depression scales were strongly correlated (Pearsons $\mathrm{r}=0.85$ ) and were therefore explored in different models. The presented model was chosen based on the log likelihood.

Figure 2 illustrates the trajectories for the three groups of interest, showing the continued fatigue group to have significantly higher levels of anxiety level, sleep disturbance, pain and pain catastrophizing in contrast to no higher level of inflammation as assessed by CRP and ultrasound PD at all visits. The main co-efficients of the mixed model analyses are shown in online supplemental table 1 . The mixed models show that the different trajectories are best differentiated by PROMs and not measures of inflammation.

\section{DISCUSSION}

The present study adds to the limited knowledge of longitudinal fatigue trajectories in patients with RA. We 
Table 1 Comparison of baseline variables across trajectories of longitudinal fatigue

\begin{tabular}{|c|c|c|c|c|c|c|}
\hline & No fatigue & $\begin{array}{l}\text { Improved } \\
\text { fatigue }\end{array}$ & $\begin{array}{l}\text { Continued } \\
\text { fatigue }\end{array}$ & $\begin{array}{l}\text { No fatigue vs } \\
\text { improved } \\
\text { fatigue }\end{array}$ & $\begin{array}{l}\text { No fatigue vs } \\
\text { continued } \\
\text { fatigue }\end{array}$ & $\begin{array}{l}\text { Improved fatigue } \\
\text { vs continued } \\
\text { fatigue }\end{array}$ \\
\hline Number & 61 & 33 & 53 & $\mathbf{p}$ & $\mathbf{p}$ & $\mathbf{p}$ \\
\hline Age (years) & $52.92(12.46)$ & $51.70(11.46)$ & 53.15 (13.59) & 0.64 & 0.92 & 0.61 \\
\hline Female gender & 44 (72.13) & $27(81.82)$ & 47 (88.68) & 0.29 & 0.03 & 0.37 \\
\hline Higher Education & 39 (63.93) & $23(67.70)$ & $22(44.00)$ & 0.30 & 0.04 & 0.02 \\
\hline Anti-CCP positive & 44 (72.13) & $26(81.25)$ & $50(96.15)$ & 0.33 & 0.001 & 0.02 \\
\hline RF positive & $32(55.17)$ & $21(72.41)$ & $38(80.85)$ & 0.12 & 0.002 & 0.39 \\
\hline Disease duration & $8.12(6.97)$ & 7.16 (7.34) & 11.71 (10.02) & 0.54 & 0.10 & 0.03 \\
\hline \multicolumn{7}{|l|}{ RA disease activity } \\
\hline Swollen joints (28) & $5.62(5.07)$ & $6.45(5.84)$ & $6.70(5.00)$ & 0.47 & 0.26 & 0.84 \\
\hline $\mathrm{CRP}(\mathrm{mg} / \mathrm{L})$ & $9.92(15.62)$ & $16.85(21.93)$ & $12.02(20.28)$ & 0.16 & 0.86 & 0.12 \\
\hline Calprotectin (mg/L) & $1.73(1.69)$ & $2.11(2.04)$ & $1.47(1.19)$ & 0.80 & 0.38 & 0.46 \\
\hline Sum PD & 14.74 (12.99) & $15.64(16.10)$ & $12.51(11.65)$ & 0.77 & 0.34 & 0.30 \\
\hline Sum GS & 31.45 (18.49) & $30.82(21.12)$ & 28.49 (16.44) & 0.88 & 0.37 & 0.57 \\
\hline \multicolumn{7}{|l|}{ PROMs } \\
\hline Patient global VAS & 27.15 (21.90) & $45.61(25.66)$ & $68.36(19.47)$ & $<0.001$ & $<0.001$ & $<0.001$ \\
\hline Tender joints (28) & $2.84(3.46)$ & $3.82(3.41)$ & $9.79(7.91)$ & $<0.19$ & $<0.001$ & $<0.001$ \\
\hline RAID sleep & $1.28(1.63)$ & $4.61(2.62)$ & $6.15(2.51)$ & $<0.001$ & $<0.001$ & 0.01 \\
\hline RAID fatigue & $1.61(1.14)$ & $5.70(1.36)$ & $7.11(1.86)$ & $<0.001$ & $<0.001$ & $<0.001$ \\
\hline Widespread pain & $4.34(2.90)$ & $6.73(4.12)$ & $8.26(5.30)$ & 0.002 & $<0.001$ & 0.16 \\
\hline HADS anxiety & $1.28(2.05)$ & $1.33(3.14)$ & $4.06(5.42)$ & 0.92 & $<0.001$ & 0.01 \\
\hline HADS depression & $0.72(1.55)$ & $0.90(2.14)$ & $3.66(4.89)$ & 0.57 & 0.004 & 0.003 \\
\hline Pain Catastrophizing & $1.18(1.02)$ & $2.57(1.44)$ & 2.89 (1.42) & $<0.001$ & $<0.001$ & 0.20 \\
\hline
\end{tabular}

Bivariate analyses No fatigue: Fatigue $\leq 3$ at baseline and at $4 / 5$ visits. Improved fatigue: Fatigue $\geq 4$ at baseline, but $\leq 3$ at 6 - and 12 -months visit. Continued: Fatigue $\geq 4$ at $5 / 6$ visits.

GS, Grey scale; HADS, Hospital anxiety and depression scale; PD, Power D oppler; VAS, Visual analogue scale. Disease duration in years.

defined and applied three different fatigue trajectories that categorise over $70 \%$ of the patients. PROMs were found to be highly significant predictors for the development of continued fatigue.

In a cohort of patients with longstanding RA we have found that 53/206 (26\%) continued to have a clinically significant fatigue after the first year of bDMARD therapy. This group was defined by a combination of a high frequency of anti-CCP and RF-positive patients in univariate analyses, and long disease duration in bivariate analyses, but there was no evidence of higher baseline inflammations compared to patients in the other fatigue trajectories.

Two systematic reviews have examined factors related to fatigue in RA. ${ }^{10}{ }^{11}$ The authors found that the level of fatigue was explained by variables related to disease activity, physical function as well as cognitive, emotional and social factors, but that the correlations between pain and fatigue were strong and persistent. Our study adds to this body of knowledge by defining baseline PROMs rather than RA disease activity as the variables that differentiated between the three trajectories.

Trajectories of fatigue in a heterogeneous cohort of RA patients have been explored by Druce et al in the Norfolk Arthritis Register. $^{12}$ By use of statistical modelling, three trajectories were described. Similar to our findings they report that those not improving were best distinguished from improvers by patientreported rather than demographic or clinical variables. Our studies differ in several ways: Firstly, the patients included in the present study have all started a bDMARD, whereas between 52 and $74 \%$ of patients in the paper by Druce et al.were treated with a DMARD. Secondly, our approach was centred around a pre-defined patient acceptable cut-off for significant fatigue at score $\geq 4 .^{9}$ Although the different instruments may not be directly compared, the cut-off is slightly lower than the cut-off for high-fatigue which has been identified as VAS $\geq 50 \mathrm{~cm}$ out of 100 defined by Pollard et $a l,{ }^{13}$ but corresponds to the level of fatigue reported in approximately $28 \%$ of patients in a large cohort of RA patients treated with a bDMARD and/or Methotexate. $^{14}$

We report that patients with continued fatigue were more frequently anti-CCP positive. This is supported by a Moroccan study, where fatigue was associated with structural damage and positivity of anti-CCP, ${ }^{15}$ but has not been mentioned in a major systematic review. ${ }^{11}$ Presently, the patients with continued fatigue had longer disease duration compared to patients in the improved fatigue 
Table 2 Baseline predictors for continued fatigue vs improved (logistic regression analyses)

\begin{tabular}{|c|c|c|}
\hline & Univariate & Multivariable \\
\hline Variables & OR $(95 \% \mathrm{Cl}) \dagger$ & OR $(95 \% \mathrm{Cl})$ \\
\hline Age (years) & $1.01(0.98-1.05)$ & $1.02(0.98-1.06)$ \\
\hline Female gender & $1.79(0.52-6.15)$ & $1.57(0.34-7.35)$ \\
\hline Higher Education & $0.30(0.11-0.81)^{*}$ & \\
\hline Anti-CCP positive & $5.51(1.02-29.60)^{\star}$ & \\
\hline RF positive & $1.72(0.56-5.29)$ & \\
\hline Disease duration & $1.03(0.98-1.10)$ & \\
\hline \multicolumn{3}{|c|}{ RA disease activity baseline } \\
\hline Swollen joints (28) & $1.01(0.92-1.10)$ & \\
\hline CRP (mg/L) & $0.99(0.97-1.01)$ & \\
\hline Calprotectin & 0.77 (0.57-1.04)d & \\
\hline Sum PD & $0.98(0.95-1.02)$ & \\
\hline Sum BM & $0.99(0.97-1.02)$ & \\
\hline \multicolumn{3}{|l|}{ PROMs } \\
\hline Patient global VAS & $1.04(1.02-1.07)^{\star \star}$ & $1.03(1.01-1.06)^{\star}$ \\
\hline Tender joints 28 & $1.21(1.08-1.35)^{*}$ & $1.19(1.04-1.35)^{\star}$ \\
\hline RAID sleep & $1.26(1.06-1.51)^{*}$ & \\
\hline Widespread pain & $1.08(0.98-1.20)$ & \\
\hline HADS anxiety & $1.17(1.03-1.33)^{\star}$ & $1.23(1.05-1.45)^{\star}$ \\
\hline HADS depression & $1.26(1.06-1.49)^{*}$ & \\
\hline Pain Catastrophizing & $1.23(0.89-1.69)$ & \\
\hline
\end{tabular}

tUnivariate model adjusted for age and gender.

ap $<0.10{ }^{*} \mathrm{p} \leq 0.05^{* *} \mathrm{p} \leq 0.001$.

No fatigue: Fatigue $\leq 3$ at baseline and at $4 / 5$ visits. Improved fatigue: Fatigue $\geq 4$ at baseline, but $\leq 3$ at 6 - and 12 -months visit. Continued: Fatigue $\geq 4$ at $5 / 6$ visits.

GS, Grey scale; HADS, Hospital anxiety and depression scale; PD, Power Doppler; VAS, Visual analogue scale.

trajectory, this is not in agreement with the findings of the same systematic review ${ }^{11}$ which concluded that disease duration did not influence fatigue.

This study presents an expansive data-collection of 6 prospective visits during a 12-month follow-up. A weakness of the study is that fatigue was only measured by a single instrument, RAID question on fatigue is however identical to the first severity question in the Bristol Numerical Fatigue Rating Scale which has been found to be reliable. ${ }^{16}$ Another weakness is that LOCF was used to impute variables and we cannot be certain that all variables were missing completely at random. However, only a very small proportion of variables were missing and all examinations were performed by the author HBF giving us some knowledge of the logistical reasons for missingness. Lastly, the study included 208 patients and the further division into trajectories posed a limitation on the number of variables that could be included in the regression models and on the statistical methods chosen to build the regression models.

The inclusion of repeated ultrasonography examinations allowed for a comprehensive assessment of
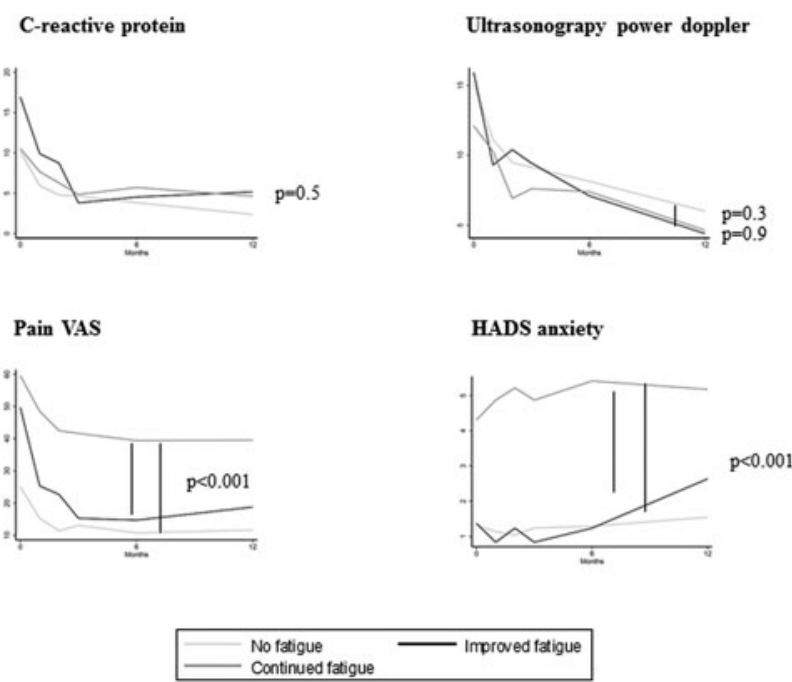

Comparison of the longitudinal course of disease activity and patient reported outcomes using mixed models via maximum bikelihood VAS; visual analogue scale, HADS; Hospital Anviety and Depression Scale.

Figure 2 Longitudinal change in disease activity and PROMS across trajectories of fatigue Comparison of the longitudinal course of disease activity and patient-reported outcomes (PROMs) using mixed models via maximum likelihood. VAS, Visual analogue scale; HADS, Hospital anxiety and depression scale.

inflammatory disease activity at all visits providing a novel and important confirmation of the lack of association between joint inflammation and fatigue, and this is a strength of the study. The cohort is a heterogeneous sample of real-life clinic and differs from cohorts of the more stringent pharmaceutical trials. This is another strength of the study. We have chosen to use a clinical rather than statistical approach to define the trajectories of interest. We believe that this strength in the identification of trajectories of fatigue in relation to a pre-defined cut-off is of relevance to both clinicians and patients. In order to categorise the maximum number of patients we allowed fatigue levels to cross the pre-defined cut-off for one out of six visits in the trajectory of no fatigue and continued fatigue and we acknowledge that this methodology is more open for individual interpretation. We show that a substantial proportion of patients still have clinically significant fatigue after 1 year of bDMARD and that the majority of patients who improve do so within the first months of bDMARD therapy.

\section{CONCLUSION}

The longitudinal course of fatigue in patients with longstanding RA who started bDMARD therapy follows one of three trajectories in the majority of patients. The development of continued fatigue was determined by PROMs and not by inflammatory RA disease activity. 
Contributors Conceived idea: SAP, HBH, TU. Data collection and planning: HBH, BM, JS. Statistical analyses: TU, HBH, SAP, JS. Writing of paper: TU, HBH, SAP, JS, BM.

Funding The authors have not declared a specific grant for this research from any funding agency in the public, commercial or not-for-profit sectors.

Competing interests None declared.

Patient consent for publication Not required.

Ethics approval The study was approved by the Regional ethical committee of south-eastern Norway.

Provenance and peer review Not commissioned; externally peer reviewed.

Data availability statement Data will be made available upon reasonable request.

Supplemental material This content has been supplied by the author(s). It has not been vetted by BMJ Publishing Group Limited (BMJ) and may not have been peerreviewed. Any opinions or recommendations discussed are solely those of the author(s) and are not endorsed by BMJ. BMJ disclaims all liability and responsibility arising from any reliance placed on the content. Where the content includes any translated material, BMJ does not warrant the accuracy and reliability of the translations (including but not limited to local regulations, clinical guidelines, terminology, drug names and drug dosages), and is not responsible for any error and/or omissions arising from translation and adaptation or otherwise.

Open access This is an open access article distributed in accordance with the Creative Commons Attribution Non Commercial (CC BY-NC 4.0) license, which permits others to distribute, remix, adapt, build upon this work non-commercially, and license their derivative works on different terms, provided the original work is properly cited, appropriate credit is given, any changes made indicated, and the use is non-commercial. See: http://creativecommons.org/licenses/by-nc/4.0/.

ORCID iDs

Sella Aarrestad Provan http://orcid.org/0000-0001-5442-902X

Brigitte Michelsen http://orcid.org/0000-0003-0103-2840

\section{REFERENCES}

1 Klareskog L, Catrina Al, Paget S. Rheumatoid arthritis. Lancet 2009;373:659-72.

2 Pilgaard T, Hagelund L, Stallknecht SE, et al. Severity of fatigue in people with rheumatoid arthritis, psoriatic arthritis and spondyloarthritis - results of a cross-sectional study. PLoS One 2019;14:e0218831.

3 Gossec L, Dougados M, Rincheval N, et al. Elaboration of the preliminary Rheumatoid Arthritis Impact of Disease (RAID) score: a EULAR initiative. Ann Rheum Dis 2009;68:1680-5.
4 Madsen LH, Ladefoged S, Corell P, et al. N-terminal pro brain natriuretic peptide predicts mortality in patients with end-stage renal disease in hemodialysis. Kidney Int 2007;71:548-54.

5 Druce KL, Bhattacharya Y, Jones GT, et al. Most patients who reach disease remission following anti-TNF therapy continue to report fatigue: results from the British Society for rheumatology biologics register for rheumatoid arthritis. Rheumatology (Oxford) 2016;55:178690.

6 Arnett FC, Edworthy SM, Bloch DA, et al. The American Rheumatism Association 1987 revised criteria for the classification of rheumatoid arthritis. Arthritis Rheum 1988;31:315-24.

7 Hammer HB, Uhlig T, Kvien TK, et al. Inflammatory assessments including ultrasound: results from a longitudinal study of rheumatoid arthritis patients. Arthritis Care Res (Hoboken) 2018;70:703-12.

8 Hammer HB, Sveinsson M, Kongtorp AK, et al. A 78-joints ultrasonographic assessment is associated with clinical assessments and is highly responsive to improvement in a longitudinal study of patients with rheumatoid arthritis starting adalimumab treatment. AnnRheumDis 2010;69:1349-51.

9 Puyraimond-Zemmour D, Etcheto A, Fautrel B, et al. Associations between five important domains of health and the patient acceptable symptom state in rheumatoid arthritis and psoriatic arthritis: a cross-sectional study of 977 patients. Arthritis Care Res (Hoboken) 2017;69:1504-9.

10 Nikolaus S, Bode C, Taal E, et al. Fatigue and factors related to fatigue in rheumatoid arthritis: a systematic review. Arthritis Care Res (Hoboken) 2013;65:1128-46.

11 Madsen SG, Danneskiold-Samsoe B, Stockmarr A, et al. Correlations between fatigue and disease duration, disease activity, and pain in patients with rheumatoid arthritis: a systematic review. Scand J Rheumatol 2016;45:255-61.

12 Druce KL, Jones GT, Macfarlane GJ, et al. The longitudinal course of fatigue in rheumatoid arthritis: results from the norfolk arthritis register J Rheumatol 2015;42:2059-65.

13 Pollard LC, Kingsley GH, Choy EH, et al. Fibromyalgic rheumatoid arthritis and disease assessment. Rheumatology (Oxford) 2010;49:924-8.

14 Olsen CL, Lie E, Kvien TK, et al. Predictors of fatigue in rheumatoid arthritis patients in remission or in a low disease activity state. Arthritis Care Res (Hoboken) 2016;68:1043-8.

15 lbn Yacoub Y, Amine B, Laatiris A, et al. Fatigue and severity of rheumatoid arthritis in Moroccan patients. Rheumatol Int 2012;32:1901-7.

16 Hewlett S, Kirwan J, Bode C, et al. The revised bristol rheumatoid arthritis fatigue measures and the rheumatoid arthritis impact of disease scale: validation in six countries. Rheumatology (Oxford) 2018;57:300-8. 